\title{
TPEF Cardiomyocyte Image 3-D Cutting and Volume Measuring based on ImageJ
}

\author{
Mengjie $\mathrm{Li}^{1}$, Shuifa Sun*1, Fangmin Dong ${ }^{1}$, Honghai Liu $^{2}$, Yonghong Shao ${ }^{3}$, \\ Bruce Z. $\mathrm{Gao}^{2}$
}

1. Institute of Intelligent Vision and Image Information, China Three Gorges University, Yichang, Hubei 443002, China

2. Department of Bioengineering, Clemson University, Clemson, SC 29635, USA

${ }^{3}$. Institute of Optoelectronics, Shenzhen University, Shenzhen, Guangdong 518060, PR China

*Corresponding author's email: watersun@ctgu.edu.cn

\begin{abstract}
Keywords: ImageJ; image-processing; Cardiomyocytes; 3D reconstruction; 3D cutting; Volume-measuring
\end{abstract}

\begin{abstract}
ImageJ is an open source image processing and analysis platform developed with Java. It can be applied to the analysis of clinical and scientific image effectively. A group of adult SD rat cardiac myocytes image using two-photon excitation fluorescence (TPEF) polarization imaging system is processed with Image J: 1)To achieve a panoramic display of the cardiomyocytes, we adopt 3D Viewer plugin to construct the 3D model; 2)Then, cutting the myocardial cell in any directions allows ones to observe the cell's internal structure; 3) With the following image processing, contrast enhancement, noise reduction, threshold segmentation, clear boundary of the object is presented; 4) Region of interest (ROI) of each slice is then calculated. The entire volume of the myocardial cell is accumulation of the product of area and scan step. The physical volume of the myocardial cell can be calculated with the relationship of physical unit and image pixel, such as pixels-per-inch (ppi).
\end{abstract}

\section{Introduction}

Image $\mathrm{J}$ is a public domain Java image processing and analysis program. It was inspired by the U. S. National Institutes of Health (NIH). With a variety of image processing and analysis functions, it can be used to help the analysis of clinical and scientific research in biomedical imaging [1]. ImageJ is widely used in the field of medical imaging diagnosis. The secondary development based on the platform is a hot topic, and there are more than 500 free plugins for the software on the Internet.

Currently, myocardial remodeling is investigated widely in the clinical research of heart disease [2]. When the mechanical loads on the heart change, cardiomyocytes undergo remodeling. During pressure and volume overload, heart will be the same as skeletal muscle, through increasing muscle mass (volume) to meet the increased workload, at this time, the addition of sarcomeres in series will lead to myocardial fibrosis (MF) [3]. When remodeling is induced by physiological means (e.g., exercise), heart function will be enhanced; however, if remodeling is generated by pathological conditions (e.g., high blood pressure and heart valve regurgitation), heart failure may be the result [4]. Studies suggested that Myofibrillogenesis Regulator 1(MR-1) was found in human myofibrils by immunohistochemistry, indicating that MR-1 is involved in hypertrophy [5]. Understanding the operational mechanisms underlying MR-1-impacts under various dynamic heart conditions is therefore of great importance for developing new treatment strategies for heart diseases [6].

TPEF polarization imaging system combined with confocal laser scanning microscopy and two-photon excitation technique, implements multi-photon nonlinear excitation by use of high photon density, has the advantages of intrinsic high-resolution and high-contrast in 3D biomedical research [7-9].

Using this imaging system, we obtained an image sequence of the sacromere structure in live cardiomyocytes isolated from adult SD rat. Through the 3D reconstruction, 3D cutting, volume-measuring and the like, initial dynamic parameters of cardiomyocytes' growth and development, even the information about MR-1 on myocardial fibrosis can be obtained. 
In the biomedical imaging field, not only is the price of medical image processing equipment expensive, but also the operation of the imaging processing requires professional and technical personnel, which makes the image processing equipment difficult to be accepted and less satisfactory. For these reasons, the technique of using PC Software-ImageJ to conduct 3D cutting and volume-measuring of myocardial cells is investigated in this paper. The rest of this paper is organized as follows. In Section II, 3D model of myocardial cells is elaborated and the cutting operation is conducted on the model. In Section III, after a serial of image processing steps, the volume of the myocardial cells is gotten. The conclusions are drawn in Section V.

\section{D myocardial cell modeling and cutting.}

Dynamic 3D map of the remodeling ventricular myocytes. When the cardiomyocyte hypertrophy occurs, the phenotype of myocardial cell changes, and the 3D construct does not all grow proportionally in all directions. Studying the structure, growth and development with 2D images has certain limitation that it does not reflect its internal structure and may even lead to erroneous results. For cardiomyocyte, 3D methods were used exclusively. 3D model can not only present its organizational structure and spatio-temporal relations accurately, but also provide the information that 2D plane analyses cann't.

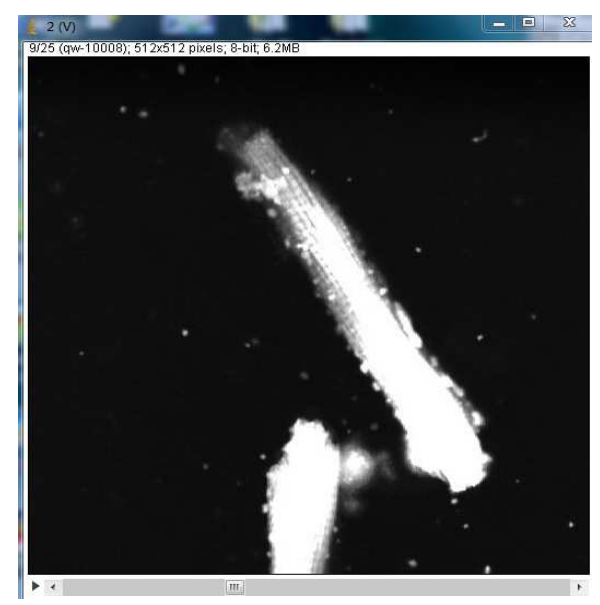

Figure 1. The original myocardial cell image stack obtained by TPEF imaging system. Interactive controls allow manual adjustments including linear translation, rotation, and magnification.

Using the TPEF imaging system, a group of adult SD rats with 25 -slice cardiac myocyte images was obtained (Figure 1). We got the dynamic model of a 3D geometry by the Z-axis continuous scanning and modeling the structure to achieve 3D panoramic display of the myocardial cells. Reconstructed 3D model could be rotated to any angle. The ImageJ monitor with its function can show any surface of the cell. Some interceptions of the surface are shown in Figure 2 (a) (b) (c) (d). As shown in Figure 2, the appearance of the structure and 3D construct of myocardial cells is obvious.

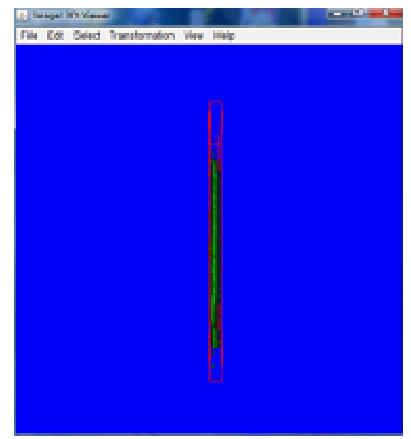

(a)

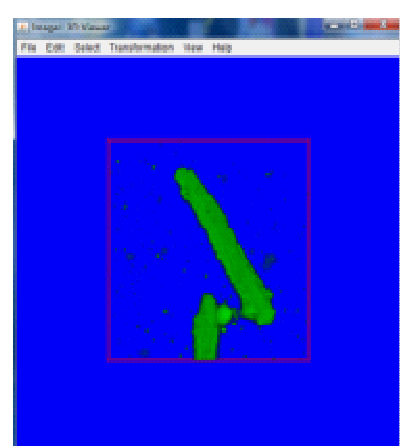

(b)

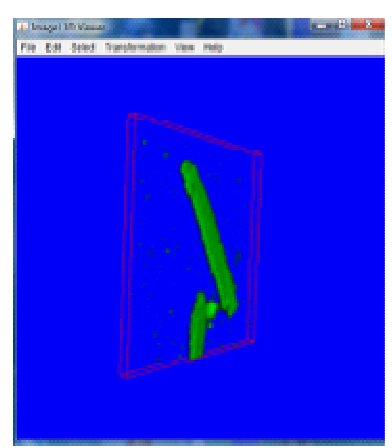

(c)

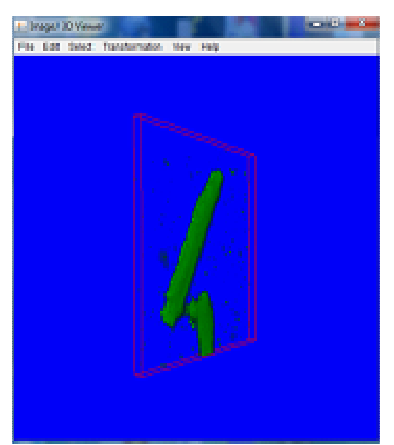

(d)

Figure 2. Examples of different views of the 3D reference model. Part (a) is a side view from which we can observe features like thickness. Part (b) is the right side of cardiomyocytes. Part (c) and (d) are other sides. ImageJ provides any side as ones like. 
3D myocardial cell cutting. With the constructed 3D model, cutting the myocardial cells allows a better visualization of internal organizational structure and connections between the myocardial cells. The cross-section of myocardial cells can be displayed at selected planes levels in the 3D model. This provides strong support to quantitative, positioning research and analyses of cells in micro-spatial structure. Combing with Z-axis consecutive image capture process of cutting cells in the $\mathrm{X}$ and $\mathrm{Y}$ planes, we get Figure 3 (a) (b). Figure 3 (c) is the Z-axis cross-sectional images obtained by $3 \mathrm{D}$ map of the horizontal cut myocardial cells. Observing these cross-sectional cell patterns, we can study the heart disease in patients with myocardial cell phenotype changes and volume increases from 3D point of view.

Figure $3(d)$ is an image of horizontal cut myocardial cell using pseudo-color enhancement, which enhances the image of subjective effects and improves the resolution of image. Comparing to the original image, the cell junction and cell adhesion molecules are more legible, a single myocardial cell is easier to identify in enhanced images [10].

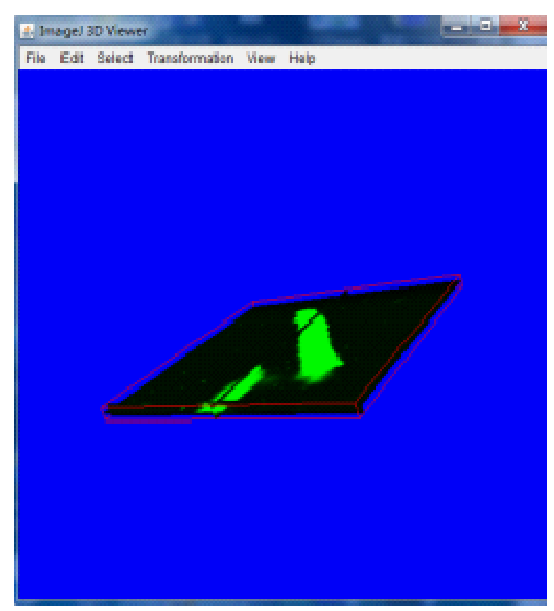

(a)

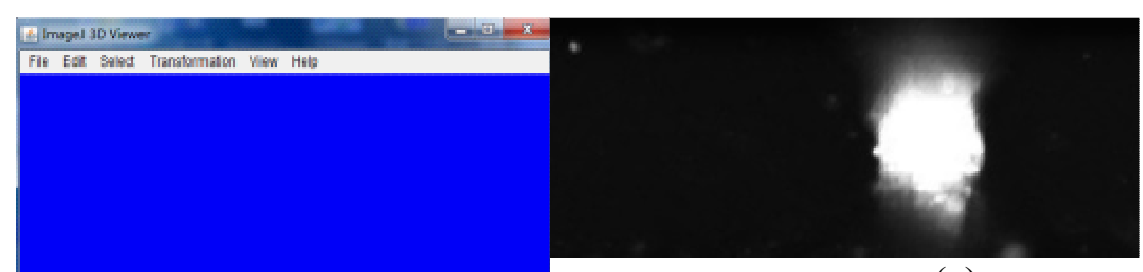

(c)

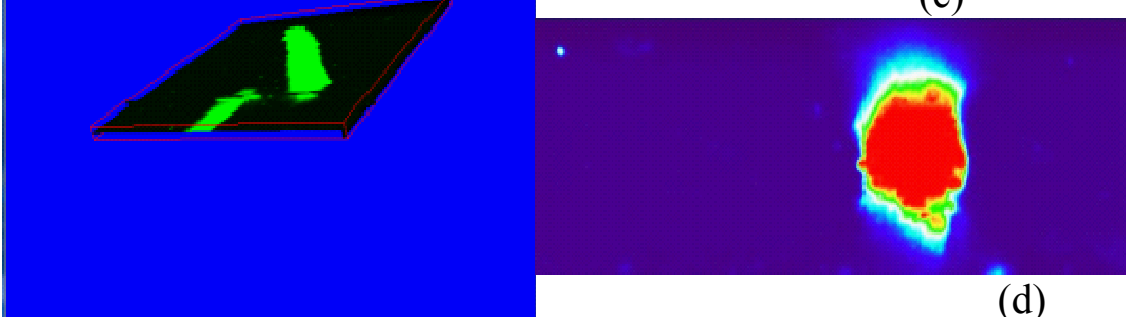

(b)

Figure 3. Cutting views in the cardiac myocyte. Parts (a-b) show the possibility to display selected images from the stack in the 3D model. Within the Z plane the images correspond to blockface. In X (part (a)) and Y (part (b)) plane, a virtual image resulting from cutting of the stack is presented in the 3D model. Part (c) is the Z-axis cross-sectional images obtained by 3D map of the horizontal cut myocardial cells. Part (d) is an image of horizontal cut myocardial cell using pseudo-color enhancement.

\section{Volume caculation of myocardial cell}

Image processing of myocardial cell slices. Generally, image processing is divided into three stages, namely, low-level image processing, image intermediate handling, advanced image processing (vision and implementation of related identification function). This paper mainly aims at the first two stages of processing, i.e. contrast enhancement, noise reduction, threshold segmentation, edge extraction.

Contrast enhancement: First, image sequences should be converted to 8 bit images (256 gray levels) and the gray value ranges from 0 to 255 . As different gray scale forms window width and window level, we can interactively adjust window width and window level to observe the different densities of myocardial cells more clearly. Second, extracting the target information and amplifying target signal can enhance contrast of the image sequence. As shown in Figure 4 (a), with the above preprocessing, the contrast between object and background is enhanced. It's helpful for later image segmentation operations.

Noise Reduction: The noise pollution in the original myocardial cell image is serious. ImageJ provides a wide range of filters for noise reduction processing, the two most common noise reduction filters of which are median filter and mean filter. The median filter response is a pixel in the image area surrounded by image-based filter to sort. The filter uses the median of the statistical sort to replace the pixel values, and it is mainly dealing with impulse noise (also known as salt and pepper noise). Mean filter replaces the value of each pixel in the image with the average gray values 
of the neighborhood pixels determined by filter mask. Its main application is to remove irrelevant details in the image, i.e. the smaller pixel area compared with the filter mask size [11]. Due to the myocardial cell images have a relatively small region of pixels, rather than salt and pepper noise, we select and use mean filter. Figure $4(\mathrm{~b})$ is the myocardial cell image after noise reduction. Assume that $(\mathrm{x}, \mathrm{y})$ is one point in the original image, then the output response to a value of $\mathrm{g}(\mathrm{x}, \mathrm{y})$ after a mean filter. Calculated as follows:

$$
g(x, y)=\frac{\sum_{s=-a}^{a} \sum_{t=-b}^{b} w(s, t) f(x+s, y+t)}{\sum_{s=-a}^{a} \sum_{t=-b}^{b} w(s, t)}
$$

Contrast to Figure 1, Figure 4 (b) is obviously found that the noise is significantly reduced after operation, but the image is relatively blurred.

Threshold segmentation and binarization: Based on distribution characteristics of the myocardial cell pixels and morphological characteristics of organization's reconstruction after divided, this paper conducts threshold segmentation. By using gray-scale histogram to analyze gray level distribution, and scan the image pixel by pixel, then mark them as the object or background to achieve image segmentation. To make the image binarization, threshold function is automatically selected. The edge detection is also quite handy when obtaining a simple image of black and white. Sobel operator sharpening can refine strong edge information. Here Sobel operator is adopted to extract edge of image based on first-order directional derivative, as shown in Figure 4 (d).

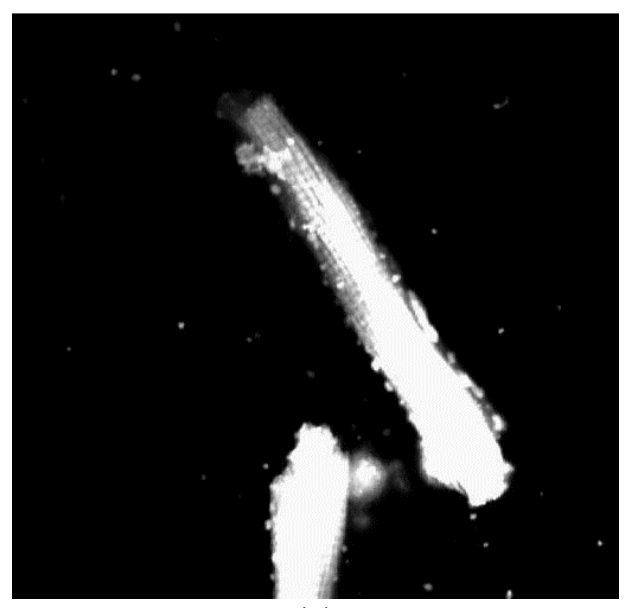

(a)

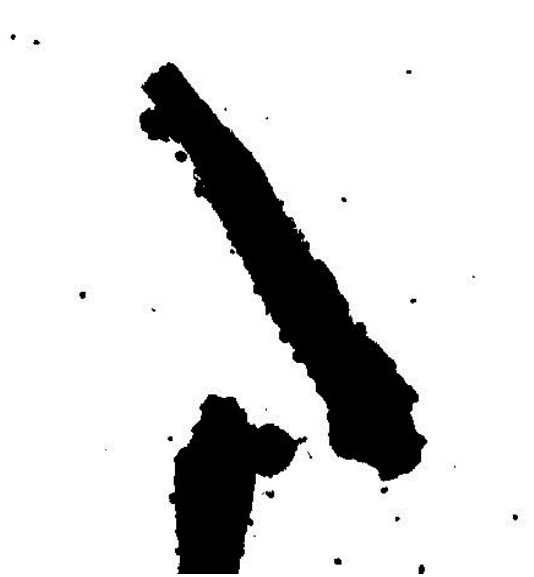

(c)

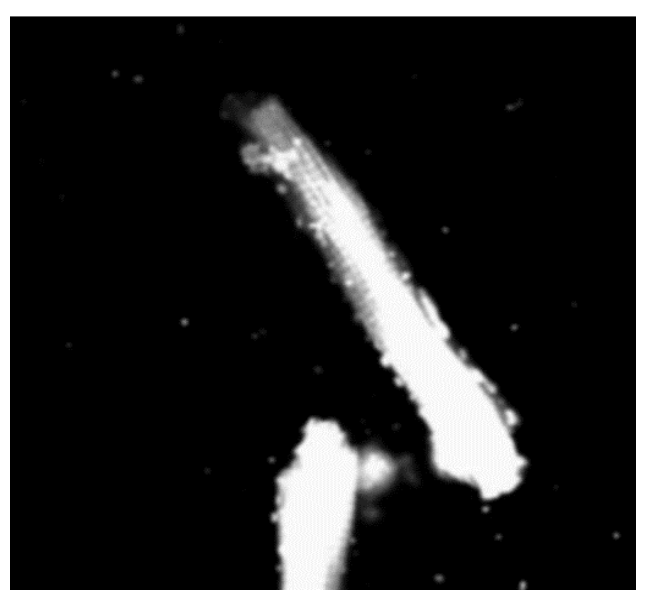

(b)

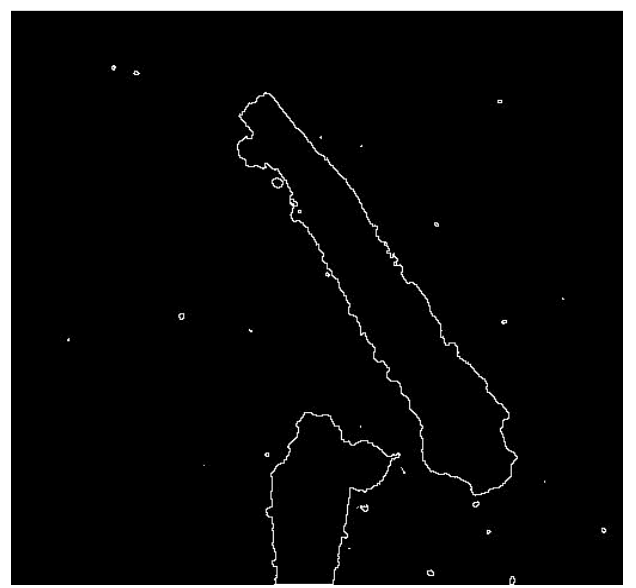

(d)

Figure 4. Image processing of myocardial cell slices. With a series of processing and stereological techniques, the later areas can be obtained exclusively. Part (a) is the myocardial cell images after conducting contrast enhancement. Part (b) is the image stack after noise reduction. Executing threshold segmentation and binarization, Part (b) can be got. Using the Sobel operator to extract edge, part (d) provides framework for date analyzing. 
Area of measurement and calculation of myocardial cells. When measuring the area of the target object in each cell image, as long as the target area is selected, area measurement function in ImageJ can be directly used. The Wand tool creates an option by describing the object in uniform color or using the object of the threshold. It traces along the edge until returning to the starting point. As shown in the image, selections are closed. Here we used the Magic Wand to select the target area and added it to the ROI Manager. But some target areas are composite region. When creating a selection, we need to hold down the Shift while clicking the Magic Wand. Then use measurement function with the method of cluster analysis, a results table will pop up, we can get the area, standard deviation, the average gray value, and skewness of ROI. If the effective image space ratio is needed to be defined, its size can be set before measurement, using standard units to replace. The results can be saved as txt, excel or other file forms are shown in Table 1:

In the table, Area stands for ROI area, Slice stands for layers. Myocardial cell image of the target area in each layer can be directly read out in the table. The units are in pixels. For example: the target area in the first layer is 1175 pixels. Through TPEF imaging system, we can obtain myocardial cells image of $512 * 512$ pixels.

Before making image area converted into the physical area of myocardial cells, we need to determine the physical size of the image. Here we only need to know the relationship between the images of physical distance and pixel, which can directly convert the pixel to actual cell area. In addition, myocardial cell is similar to the oval. When scanned to the later few slices, owing to the binarization threshold selection and other problems, the target area is 0 .

Table 1 Results of each slice area

\begin{tabular}{|c|c|c|c|c|c|c|c|c|c|}
\hline Slice & $\mathbf{1}$ & $\mathbf{2}$ & $\mathbf{3}$ & $\mathbf{4}$ & $\mathbf{5}$ & $\mathbf{6}$ & $\mathbf{7}$ & $\mathbf{8}$ & $\mathbf{9}$ \\
\hline Area & 1175 & 1551 & 1834 & 4080 & 5584 & 9179 & 10471 & 15921 & 20187 \\
\hline Slice & $\mathbf{1 0}$ & $\mathbf{1 1}$ & $\mathbf{1 2}$ & $\mathbf{1 3}$ & $\mathbf{1 4}$ & $\mathbf{1 5}$ & $\mathbf{1 6}$ & $\mathbf{1 7}$ & $\mathbf{1 8}$ \\
\hline Area & $\mathbf{2 2 3 1 5}$ & 23506 & 24037 & 23883 & 23354 & 22416 & 19857 & 19001 & 15207 \\
\hline Slice & $\mathbf{1 9}$ & $\mathbf{2 0}$ & $\mathbf{2 1}$ & $\mathbf{2 2}$ & $\mathbf{2 3}$ & $\mathbf{2 4}$ & $\mathbf{2 5}$ & & \\
\hline Area & 11503 & $\mathbf{6 2} 71$ & 1334 & 0 & 0 & 0 & 0 & & \\
\hline
\end{tabular}

\section{Volume of the myocardial cells}

The specific ideas of cumulative method calculated the volume of myocardial cells is: we should calculate the volume of myocardial cells is: we should calculate the slice area of Si first, and each image is regarded as a layer of thin slices in 3D segmentation, then measured area Si is multiplied by the thickness $\mathrm{d}$ and the cumulative sum is the volume of the myocardial cells, the following formula:

$$
V=\sum_{i=1}^{n} S_{i}^{*} d
$$

$\mathrm{V}$ is the volume of myocardial cells, and $\mathrm{i}$ is the layer of the image area $(\mathrm{n}=25)$, $\mathrm{d}$ is the thickness. Cumulative method is similar to the integral principle. The error depends mainly on the scan interval size and the accuracy of select the target object region. As the scanning layer interval of the myocardial cells is small, ImageJ can simultaneously display several selected area or ROI. Besides, though the function approximation method and complex interpolation method can also be used to calculate the myocardial cell size, taking into account the calculation load and the poor stability, the simple, little error cumulative method is adopted here

\section{Summary}

ImageJ is very small, only $4 \mathrm{~KB}$ memory space, but a powerful utility. It can be run on variant platforms, such as the Microsoft Windows, Mac OS and Linux. A variety of medical image formats, such as DICOM, are supported It can calculate the area and pixel value the user-specified area, but also can measure the distance and angle. It supports low-level image processing, the intermediate 
image processing and advanced image processing. ImageJ's design uses an open architecture, Java plug-in extensions. ImageJ can be independent of vast medical instruments and besides fast. While the course of studying myocardial cells is often elaborate and complex. Applying ImageJ to the research of myocardial cells during growth and development can avoid a mass of heavy work. In order to get the heart disease information, this paper presents research approach to study the cardiac remodeling process, explore MF. However, only using the myocardial images obtained by the TPEF imaging system to investigate the MR-1 is not comprehensive enough. Combining with second harmonic generation (SHG) images to study the myocardial cells will be the future work [4].

\section{Acknowledgment}

This project is supported by National Natural Science Foundation of China (60972162, 61102155, 31171372), Outstanding Young and Middle-aged Innovative Research Team Plan of Hubei Province of China (T201002), the Young and Middle-aged Science Funding of Hubei Provincial Department of Education (Q20111205).

\section{References}

[1] Michael Double, Michal M Klosowski, Ignacio Arganda-Carreras, Fabrice P Cordelieres, Robert P Dougherty, Jonathan S Jackson, Benjamin Schmid, John R Hutchinson, and Sandra J Shefelbine. Bone J: Free and extensible bone image analysis in ImageJ. Bone, 47(6): 1076-9, Dce 2010 .

[2] Dixon Ian MC, Ju H, Reid NL, et al. Cardiac collagen remodeling in the cardiomyopathic Syrian hamster and the effect of Losarton. J Mol Cell Cardiol, 1997, 29: 1837-1850

[3] Sun Y. Local angiotension II and myocardial fibrosis. Adv Exp Med Biol, 1997, 432: 5561.

[4] Yonghong Shao, Honghai Liu, Tong Ye. 3D Myofibril Imaging in Live Cardiomyocytes via Hybrid SHG-TPEF Microscopy. SPIE Digital Library, 2011, 1-6.

[5] Li TB, Liu XH, Feng S et al. Characterization of MR-1, a novel myofibrillogenesis regulator in human muscle. Acta Biochimica et Biophysica Sinica 2004; 36(6): 412-418.

[6] Vivian S, Michael T, Neil M, et al. Hepatic MR imaging with a dynamic contrast-enhanced isotropic volumetric interpolated breath-hold examination: feasibility, reproducibility, and technical quality. Radiologic, 2000, 215(1): 365-372.

[7] M.Oheim, D. J. Michael, M. Geisbauer, et al. Principles of two-photon excitation fluorescence microscopy and other nonlinear imaging approaches. Advanced Drug Delivery Reviews, 2006,58:788-808.

[8] G.E.Stutzmann, I. Parker. Dynamic multiphoton imaging: a live view from cells to systems. Methods, 2003, 30: 3-15.

[9] A.Diaspro,M.Robello. Two-photon excitation of fluorescene for three-dimensional optical imaging of biologic structures. Journal of Photochemistry and Photobiology B: Biology, 2000, 55(1): $1-8$.

[10]Sheng Zhong, Xiaoyu Jiang, Zhen wei. Pseudo-color Coding with Phase-modulated Image Density. Proceedings of International Conferenceon Micro/ Nano Optical Engineering(ICOME), 2011, 130-131.

[11]R.C. Gonzalez and R.E. Woods, Digital Image Processing, Prentice-Hall, 2002. 